TRANSACTIONS OF THE

AMERICAN MATHEMATICAL SOCIETY

Volume 356, Number 8, Pages 3167-3187

S 0002-9947(03)03487-1

Article electronically published on October 29, 2003

\title{
BOUNDARY CORRESPONDENCE OF NEVANLINNA COUNTING FUNCTIONS FOR SELF-MAPS OF THE UNIT DISC
}

\author{
PEKKA J. NIEMINEN AND EERO SAKSMAN
}

\begin{abstract}
Let $\phi$ be a holomorphic self-map of the unit disc $\mathbb{D}$. For every $\alpha \in$ $\partial \mathbb{D}$, there is a measure $\tau_{\alpha}$ on $\partial \mathbb{D}$ (sometimes called Aleksandrov measure) defined by the Poisson representation $\operatorname{Re}(\alpha+\phi(z)) /(\alpha-\phi(z))=\int P(z, \zeta) d \tau_{\alpha}(\zeta)$. Its singular part $\sigma_{\alpha}$ measures in a natural way the "affinity" of $\phi$ for the boundary value $\alpha$. The affinity for values $w$ inside $\mathbb{D}$ is provided by the Nevanlinna counting function $N(w)$ of $\phi$. We introduce a natural measure-valued refinement $M_{w}$ of $N(w)$ and establish that the measures $\left\{\sigma_{\alpha}\right\}_{\alpha \in \partial \mathbb{D}}$ are obtained as boundary values of the refined Nevanlinna counting function $M$. More precisely, we prove that $\sigma_{\alpha}$ is the weak* limit of $M_{w}$ whenever $w$ converges to $\alpha$ non-tangentially outside a small exceptional set $E$. We obtain a sharp estimate for the size of $E$ in the sense of capacity.
\end{abstract}

\section{INTRODUCTION}

Let $\phi$ be a non-constant holomorphic self-map of the unit disc $\mathbb{D}$. Its Nevanlinna counting function is defined for all $w \in \mathbb{D} \backslash\{\phi(0)\}$ by

$$
N(w)=\sum_{z \in \phi^{-1}\{w\}}-\log |z|,
$$

where each pre-image is counted according to its multiplicity and an empty sum is regarded as zero. The function $N$ provides a measure of how often and how strongly $\phi$ assumes each of the values in $\mathbb{D}$. The concept is due to R. Nevanlinna, who introduced the "Anzahlfunktion" as a central tool for the value distribution theory of entire and meromorphic functions (see $[\mathrm{N}]$ ). Of other classical contributions, specialized to the case of bounded functions, we mention the majorant inequalities due to Littlewood [Li1], Li2] and Lehto [L. More recently, starting with the work of J. H. Shapiro [S1], counting function methods have also played an important role in the study of composition operators on various function spaces.

The function $\phi$ also determines a family of positive measures $\left\{\tau_{\alpha}\right\}_{\alpha \in \partial \mathbb{D}}$, defined on the unit circle $\partial \mathbb{D}$ by means of the Poisson representation formula

$$
\operatorname{Re} \frac{\alpha+\phi(z)}{\alpha-\phi(z)}=\int_{\partial \mathbb{D}} P(z, \zeta) d \tau_{\alpha}(\zeta)
$$

Received by the editors February 3, 2003.

2000 Mathematics Subject Classification. Primary 30D35, 30D50; Secondary 47B33.

Key words and phrases. Nevanlinna counting function, Aleksandrov measure, multiplicity, boundary value, angular derivative.

The first author was supported by the Academy of Finland, project 49077. 
These measures have proven useful in several branches of modern function theory. Let us only refer to their role in the theory of so-called de Branges-Rovnyak spaces (see e.g. [Sa2]) and in the investigation of compactness properties of composition operators ([Sa1], $[\mathrm{CM}], \underline{\mathrm{Sh}}])$. Heuristically speaking, $\tau_{\alpha}$ can be seen as measuring how strong an "affinity" (and at which points) the function $\phi$ has for the boundary value $\alpha$, and of particular interest is its singular part, which we will denote by $\sigma_{\alpha}$. For the sake of illustration, one may consider the special case when $\phi$ extends holomorphically across the unit circle $\partial \mathbb{D}$ : it is then not difficult to see that $\tau_{\alpha}$ has an atom at a point $\zeta \in \partial \mathbb{D}$ if and only if $\phi(\zeta)=\alpha$, and in this case $\tau_{\alpha}(\{\zeta\})=1 /\left|\phi^{\prime}(\zeta)\right|$. In A] A. B. Aleksandrov made explicit use of the measures $\tau_{\alpha}$ as he studied the multiplicity and other properties of boundary values of inner functions. For this reason $\tau_{\alpha}$ is sometimes called the Aleksandrov measure of $\phi$ at the point $\alpha$, and we will follow that practice here.

Since $N$ measures the affinity of $\phi$ for values in the unit disc and $\tau_{\alpha}$ 's do the same for values on its boundary, it seems natural to ask if there is any relation between $\tau_{\alpha}$ and the boundary behaviour of $N(w)$ as $w \rightarrow \alpha$. A clear hint in this direction comes from the papers by Shapiro [S1] and Cima and Matheson [CM], which provide two different expressions for the essential norm of the composition operator $f \mapsto f \circ \phi$ on the Hardy space $H^{2}$. Combining these expressions yields the identity

$$
\limsup _{|w| \rightarrow 1-} \frac{N(w)}{-\log |w|}=\sup _{\alpha \in \partial \mathbb{D}}\left\|\sigma_{\alpha}\right\|,
$$

indicating that the maximal size of the singular parts of the Aleksandrov measures is related to the decay rate of the Nevanlinna counting function at the boundary.

The present paper provides a far-reaching extension of (1.1). In fact, we will establish a definite local boundary correspondence between the two measures of affinity involved. To this end we introduce a natural measure-valued (and normalized) refinement of the Nevanlinna counting function of $\phi$ by defining, for every $w \in \mathbb{D} \backslash\{0\}$,

$$
M_{w}=\sum_{z \in \phi^{-1}\{w\}} \frac{-\log |z|}{-\log |w|} \delta_{z}
$$

where each pre-image $z$ is counted according to its multiplicity and $\delta_{z}$ is the unit mass (i.e. Dirac measure) at $z$. It seems appropriate to call these measures the Nevanlinna counting measures of $\phi$. Note that $\left\|M_{w}\right\|=N(w) /(-\log |w|)$.

The following theorem is our main result. Here we let $\Gamma(\alpha, \rho)$ denote the nontangential approach region obtained by taking the interior of the convex hull of a point $\alpha \in \partial \mathbb{D}$ and a disc $B(0, \rho)$ with $0<\rho<1$.

Theorem 1.1. Let $\Gamma=\Gamma(\alpha, \rho)$ be an arbitrary non-tangential approach region at a point $\alpha \in \partial \mathbb{D}$. Then there exists a set $E \subset \Gamma$ of finite Green capacity such that

$$
M_{w} \rightarrow \sigma_{\alpha} \text { weak }^{*} \quad \text { as } w \rightarrow \alpha \text { in } \Gamma \backslash E,
$$

where $M_{w}$ and $\sigma_{\alpha}$ are regarded as measures on $\overline{\mathbb{D}}$. In other words, for every continuous function $f$ on $\overline{\mathbb{D}}$, we have

$$
\int_{\mathbb{D}} f d M_{w} \rightarrow \int_{\partial \mathbb{D}} f d \sigma_{\alpha} \quad \text { as } w \rightarrow \alpha \text { in } \Gamma \backslash E .
$$


The concept of Green capacity will be discussed later; at this point we only remark that the capacity of a set $E \subset \Gamma(\alpha, \rho)$ is finite if and only if the capacity of $E \cap B(\alpha, r)$ decreases to zero as $r \rightarrow 0+$. This also implies that the ordinary logarithmic capacity of the scaled set $\frac{1}{r}(E \cap B(\alpha, r))$ tends to zero as $r \rightarrow 0+$. As a consequence, it is always possible to find sequences $\left(w_{n}\right)$ tending to $\alpha$ radially such that $M_{w_{n}} \rightarrow \sigma_{\alpha}$ weak*. Observe also that because the measures involved are positive, the weak* ${ }^{*}$ convergence implies the convergence of total masses.

In Section 8 we will construct examples showing that the capacity estimate in Theorem 1.1 is the best possible. In particular, it is not always true that $M_{r \alpha} \rightarrow$

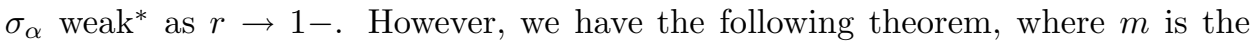
normalized Lebesgue measure on the unit circle:

Theorem 1.2. For every continuous function $g$ on $\partial \mathbb{D} \times \overline{\mathbb{D}}$,

$$
\lim _{r \rightarrow 1-} \int_{\partial \mathbb{D}}\left[\int_{\mathbb{D}} g(\alpha, z) d M_{r \alpha}(z)\right] d m(\alpha)=\int_{\partial \mathbb{D}}\left[\int_{\partial \mathbb{D}} g(\alpha, z) d \sigma_{\alpha}(z)\right] d m(\alpha) .
$$

Let us emphasize here that the preceding two theorems can be regarded as purely function-theoretic statements providing a link between the value distribution theory inside the disc and on its boundary. However, Theorem 1.1 is also of operatortheoretic significance as it explains the rather mysterious identity (1.1) resulting from the two norm expressions for the induced composition operator. A substantial part of our proof actually employs techniques coming from the theory of composition operators.

This paper is organized as follows. Sections 2 to 4 contain some preliminary material on Aleksandrov measures, Nevanlinna counting function and Green capacity. The proof of Theorem [1.1 is carried out in Sections 5] to 7 The outline of the argument is as follows: We first observe (Lemma [5.1) that the limiting mass of $M_{w}$ never exceeds the mass of $\sigma_{\alpha}$, that is,

$$
\limsup _{w \rightarrow \alpha} \frac{N(w)}{-\log |w|} \leq\left\|\sigma_{\alpha}\right\|
$$

The essence of our argument then consists of determining under what kind of restrictions on $w$ the desired limit $\left\|\sigma_{\alpha}\right\|$ is attained. We show (Proposition 6.1) that this occurs as $w \rightarrow \alpha$ in $\Gamma \backslash E$, where $\Gamma$ and $E$ are as in the statement of the theorem. Finally we complete the proof by showing (Proposition 7.1) that, surprisingly enough, the convergence of total masses automatically guarantees the weak* convergence of measures.

In Section 8 we proceed to discussing the optimality of Theorem 1.1. The proof of Theorem 1.2 occupies Section 9 and finally some remarks on the relation of our work to angular derivatives are recorded in Section 10.

\section{PRELIMINARIES}

\section{Aleksandrov measures}

We assume throughout this paper that $\phi$ is a non-constant holomorphic self-map of the unit disc $\mathbb{D}$. We also denote by $m$ the Lebesgue measure on the unit circle $\partial \mathbb{D}$, normalized to have unit total mass. 
Let us recall for convenience that the Aleksandrov measures of $\phi$ are defined for every $\alpha \in \partial \mathbb{D}$ by the Poisson representation formula

$$
\operatorname{Re} \frac{\alpha+\phi(z)}{\alpha-\phi(z)}=\frac{1-|\phi(z)|^{2}}{|\alpha-\phi(z)|^{2}}=\int_{\partial \mathbb{D}} P(z, \zeta) d \tau_{\alpha}(\zeta),
$$

where $P(z, \zeta)=\frac{1-|z|^{2}}{|\zeta-z|^{2}}$ is the Poisson kernel. We also let $d \tau_{\alpha}=h_{\alpha} d m+d \sigma_{\alpha}$ be the Lebesgue decomposition of $\tau_{\alpha}$, so that $h_{\alpha} \in L^{1}(m)$ and $\sigma_{\alpha}$ is singular. The following are some of the basic properties of Aleksandrov measures (cf. [Sh]):

(a) The absolutely continuous part of $\tau_{\alpha}$ is given by

$$
h_{\alpha}(\zeta)=\frac{1-\left|\phi^{*}(\zeta)\right|^{2}}{\left|\alpha-\phi^{*}(\zeta)\right|^{2}}
$$

where $\phi^{*}(\zeta)$ are the non-tangential boundary limits of $\phi$ (defined and different from $\alpha$ for a.e. $\zeta \in \partial \mathbb{D})$.

(b) The measure $\tau_{\alpha}$ is singular if and only if $\phi$ is an inner function, i.e. $\left|\phi^{*}(\zeta)\right|=1$ for almost every $\zeta \in \partial \mathbb{D}$.

(c) The total mass of the singular part of $\tau_{\alpha}$ is

$$
\left\|\sigma_{\alpha}\right\|=\frac{1-|\phi(0)|^{2}}{|\alpha-\phi(0)|^{2}}-\int_{\partial \mathbb{D}} \frac{1-\left|\phi^{*}(\zeta)\right|^{2}}{\left|\alpha-\phi^{*}(\zeta)\right|^{2}} d m(\zeta) .
$$

(d) For $\sigma_{\alpha}$-a.e. $\zeta \in \partial \mathbb{D}$, we have $\phi^{*}(\zeta)=\alpha$.

(e) The measures $\tau_{\alpha}$ and $\sigma_{\alpha}$ have an atom at a point $\zeta \in \partial \mathbb{D}$ if and only if $\phi^{*}(\zeta)=$ $\alpha$ and $\phi$ has a finite angular derivative at $\zeta$ in the sense of Carathéodory. In this case $\tau_{\alpha}(\{\zeta\})=1 /\left|\phi^{\prime}(\zeta)\right|$.

Facts (a)-(d) are consequences of the basic properties of Poisson integral representations (see e.g. [R2, Chapter 11]). We will say more about angular derivatives and fact (e) in Section 10 .

Finally, we should remark that all finite positive measures occur as Aleksandrov measures of some $\phi$. Indeed, if $\mu$ is a given measure of this type, one may use the Herglotz integral formula

$$
\frac{1+\phi(z)}{1-\phi(z)}=\int_{\partial \mathbb{D}} \frac{\zeta+z}{\zeta-z} d \mu(\zeta)
$$

to obtain $\phi$ whose Aleksandrov measure at the point 1 is $\mu$.

\section{NeVAnLinna COUNTING FUnCtion}

This section collects some well-known properties of the Nevanlinna counting function that will be needed in the sequel. For more detailed expositions we refer to $\mathrm{S1}$ and [S2]. Let us recall here that the Nevanlinna counting function of $\phi$ is defined on the set $\mathbb{D} \backslash\{\phi(0)\}$ by the formula

$$
N(w)=\sum_{z \in \phi^{-1}\{w\}}-\log |z| .
$$

To indicate its dependence on $\phi$ we may sometimes write $N_{\phi}$ instead of $N$. 
3.1. Integral representation and subharmonicity. In order to examine the functional properties of $N$, it is useful to rewrite it in terms of an integral representation. Define, for each $w \in \mathbb{D}$, the Frostman transform of $\phi$ by

$$
\phi_{w}(z)=\frac{w-\phi(z)}{1-\bar{w} \phi(z)} .
$$

Note that $\phi_{w}=\psi_{w} \circ \phi$, where $\psi_{w}(z)=(w-z) /(1-\bar{w} z)$ is a self-inverse conformal automorphism of $\mathbb{D}$. On applying Jensen's formula [R2 15.18] to $\phi_{w}$ one can now easily show that

$$
N(w)=\lim _{r \rightarrow 1-} \int_{\partial \mathbb{D}} \log \left|\phi_{w}(r \zeta)\right| d m(\zeta)-\log \left|\phi_{w}(0)\right| .
$$

Suggested by the above expression we also consider the function

$$
\bar{N}(w)=\int_{\partial \mathbb{D}} \log \left|\phi_{w}^{*}(\zeta)\right| d m(\zeta)-\log \left|\phi_{w}(0)\right| .
$$

By Fatou's lemma, $N \leq \bar{N}$ at every point of $\mathbb{D}$. Furthermore, a result of Rudin [R1] implies that $N(w)$ and $\bar{N}(w)$ are equal for quasi-every (q.e.) $w \in \mathbb{D}$, i.e. outside an exceptional set of capacity zero. In fact, it is easy to see that equality occurs if and only if there is no singular inner factor in the canonical factorization of $\phi_{w}$, that is, if $\phi_{w}$ factorizes to a Blaschke product and an outer function.

It is important to note that the function $\bar{N}$ is subharmonic in the unit disc, except for a logarithmic pole at $\phi(0)$. To see this, let $\mu$ be the distribution of the boundary values of $\phi$, i.e. $\mu(A)=m\left(\left\{\zeta \in \partial \mathbb{D}: \phi^{*}(\zeta) \in A\right\}\right)$ for every Borel set $A \subset \overline{\mathbb{D}}$. Then the definition of $\bar{N}$ can be rewritten as

$$
\bar{N}(w)=-G_{\mu}(w)+\log \left|\frac{1-\overline{\phi(0)} w}{w-\phi(0)}\right|,
$$

where $G_{\mu}$ is the Green potential associated to $\mu$ (see Section 4 below) and the last term is harmonic in $\mathbb{D} \backslash\{\phi(0)\}$.

3.2. Littlewood's inequality. Since the integrand on the right-hand side of (3.1) is negative, one obtains the well-known Inequality of Littlewood [Li1] (see also [L]):

$$
\bar{N}(w) \leq-\log \left|\phi_{w}(0)\right|=\log \left|\frac{1-\bar{w} \phi(0)}{w-\phi(0)}\right| .
$$

This clearly yields the general estimate $O(-\log |w|)$ for the rate of decay of $\bar{N}(w)$ and $N(w)$ as $|w| \rightarrow 1-$. Obviously, equality occurs in (3.2) for any (and hence all) $w \in \mathbb{D}$ if and only if $\phi$ is an inner function. In that case the result of Rudin cited above reduces to a celebrated theorem of Frostman [Fr] asserting that the transforms $\phi_{w}$ are Blaschke products for q.e. $w \in \mathbb{D}$.

\section{Green capacity}

4.1. Definition. The concept of Green capacity is similar to that of logarithmic capacity, which is discussed in many textbooks (e.g. [F], [Ra]). The only real difference is that the logarithmic kernel $-\log |z-w|$ is replaced by the Green function

$$
g(z, w)=\log \left|\frac{1-z \bar{w}}{z-w}\right|=-\log \rho(z, w) .
$$

Here $\rho(z, w)=|z-w| /|1-z \bar{w}|$ is the pseudo-hyperbolic metric for $\mathbb{D}$. 
One of the commonest ways to define the Green capacity as well as the logarithmic capacity relies on least-energy considerations. If $\mu$ is a positive Borel measure on $\mathbb{D}$, define the energy integral

$$
I(\mu)=\int G_{\mu}(z) d \mu(z)=\iint g(z, w) d \mu(w) d \mu(z),
$$

where the function $G_{\mu}(z)=\int g(z, w) d \mu(w)$ is the Green potential associated to $\mu$. Now let $E$ be an arbitrary subset of $\mathbb{D}$ and put

$$
V(E)=\inf I(\nu),
$$

where the infimum is taken over all Borel probability measures $\nu$ that are supported on compact subsets of $E$. Then the Green capacity of $E$ is defined to be the nonnegative (possibly infinite) number

$$
C(E)=1 / V(E) .
$$

Since the pseudo-hyperbolic metric is invariant under conformal automorphisms of the disc, the same is true of the Green capacity. One should also note that the Green capacity and logarithmic capacity determine the same polar sets (i.e. sets of capacity zero) in $\mathbb{D}$ because the associated kernel functions are comparable on compact subsets of the unit disc. Moreover, it is well known that Borel polar sets have Lebesgue measure zero, and this is actually true of their $s$-dimensional Hausdorff measure for every $s>0$.

4.2. Equilibrium measures. If $K$ is a compact subset of $\mathbb{D}$, it can be shown (cf. [Ra, Sec. 3.3]) that there exists a Borel probability measure $\nu_{0}$ supported on the boundary of $K$ such that $I\left(\nu_{0}\right)=V(K)$. In addition, the associated Green potential $G_{\nu_{0}}$ is bounded above by $V(K)$ everywhere on $\mathbb{D}$ and it is equal to $V(K)$ on $K$ except for a polar set contained in the boundary of $K$. Such a measure $\nu_{0}$ is called an equilibrium measure of $K$, and it is unique if $C(K)>0$. Often we will find it more convenient to work with the measure $\mu_{0}=C(K) \nu_{0}$, which is uniquely determined by $K$ and called the capacitary distribution of $K$.

The existence of equilibrium measures entails alternative expressions for the Green capacity. The proof of the next lemma is standard but included here for the convenience of the reader.

Lemma 4.1. If $K$ is a compact subset of $\mathbb{D}$, then

$$
\begin{aligned}
C(K) & =\sup \left\{\|\mu\|: \mu \in M(K), G_{\mu} \leq 1 \text { on } \mathbb{D}\right\} \\
& =\inf \left\{\|\mu\|: \mu \in M(\mathbb{D}), G_{\mu} \geq 1 \text { q.e. on } K\right\},
\end{aligned}
$$

where $M(K)$ and $M(\mathbb{D})$ are the sets of all positive Borel measures supported on $K$ and $\mathbb{D}$, respectively.

Proof. Let us denote the supremum by $S$ and the infimum by $s$. Note, first of all, that if $\mu_{0}$ is the capacitary distribution of $K$, then $\left\|\mu_{0}\right\|=C(K)$ and $G_{\mu_{0}} \leq 1$ on $\mathbb{D}$ with equality quasi-everywhere on $K$. Hence clearly $s \leq C(K) \leq S$.

Now let $\mu \in M(K)$ be an arbitrary non-zero measure such that $G_{\mu} \leq 1$ on $\mathbb{D}$. Then the probability measure $\nu=\|\mu\|^{-1} \mu$ satisfies $G_{\nu} \leq\|\mu\|^{-1}$ on $\mathbb{D}$ and hence $V(K) \leq I(\nu) \leq\|\mu\|^{-1}$. Thus $\|\mu\| \leq C(K)$, and we conclude that $S \leq C(K)$.

Finally let $\mu \in M(\mathbb{D})$ be an arbitary measure such that $G_{\mu} \geq 1$ quasi-everywhere on $K$. It is not difficult to see that the capacitary distribution $\mu_{0}$ gives measure 
zero to Borel sets of capacity zero. Thus, by Fubini's theorem,

$$
\|\mu\| \geq \int G_{\mu_{0}} d \mu=\int G_{\mu} d \mu_{0} \geq\left\|\mu_{0}\right\|=C(K) .
$$

Hence $s \geq C(K)$.

4.3. Capacity of pseudo-hyperbolic discs. The Green capacities of closed discs in $\mathbb{D}$ can be computed explicitly in terms of their pseudo-hyperbolic radii. First consider the closed Euclidean disc $\bar{B}(0, r)$ centred at the origin with radius $r \in] 0,1[$. The corresponding equilibrium measure $\nu_{0}$ is the uniform probability measure on the circle $|z|=r$. Thus,

$$
G_{\nu_{0}}(z)=\int_{\partial \mathbb{D}} g(z, r \zeta) d m(\zeta)=-\int_{\partial \mathbb{D}} \log |z-r \zeta| d m(\zeta)=-\log (r \vee|z|)
$$

In particular, the Green capacity of $\bar{B}(0, r)$ is $(-\log r)^{-1}$. By applying the conformal invariance of the Green capacity we now see that the capacity of any closed pseudo-hyperbolic disc

$$
\bar{D}(a, r)=\{z \in \mathbb{D}: \rho(z, a) \leq r\}
$$

(where $a \in \mathbb{D}$ and $0<r<1$ ) is equal to $(-\log r)^{-1}$. Furthermore, the associated equilibrium measure $\nu_{0}$ satisfies

$$
G_{\nu_{0}}(z)= \begin{cases}-\log r, & z \in \bar{D}(a, r), \\ -\log \rho(z, a), & z \notin \bar{D}(a, r) .\end{cases}
$$

4.4. Asymptotic additivity. Taking advantage of Lemma 4.1 and the existence of capacitary distributions, one can show that the Green capacity is an outer measure (i.e. countably subadditive set function) on the Borel subsets of the unit disc. Although it is, in general, highly non-additive, the following "asymptotic" additivity property holds true: whenever $K_{j}(j=1,2, \ldots)$ are compact sets in $\mathbb{D}$ with fixed capacities $c_{j}$, respectively, and $d=\inf \left\{\rho\left(K_{j}, K_{k}\right): j \neq k\right\}$, then

$$
C\left(\bigcup_{j=1}^{\infty} K_{j}\right) \rightarrow \sum_{j=1}^{\infty} c_{j} \quad \text { as } d \rightarrow 1-\text {. }
$$

To sketch a proof, we fix any $n \geq 1$ and let $\mu$ be the sum of the capacitary distributions of the sets $K_{1}, \ldots, K_{n}$. Then $G_{\mu} \leq 1-(\log d) \sum_{j=1}^{n} c_{j}$, so an application of Lemma 4.1 yields

$$
C\left(\bigcup_{j=1}^{\infty} K_{j}\right) \geq \frac{\sum_{j=1}^{n} c_{j}}{1-(\log d) \sum_{j=1}^{n} c_{j}} .
$$

Notice that the right-hand side here tends to $\sum_{j=1}^{n} c_{j}$ as $d \rightarrow 1-$. Since $n$ was arbitary, the desired conclusion follows from this and the subadditivity of $C$.

\section{PROOF OF THEOREM 1.1}

\section{Preservation of mass along a sequence}

We now turn our attention to the proof of Theorem 1.1 by investigating the convergence of the total mass of the Nevanlinna counting measures to that of the singular parts of Aleksandrov measures. The present section contains some preliminary observations, and the main argument will be carried out in the next section. 
Our first lemma observes that the limiting mass of $M_{w}$ never exceeds the mass of $\sigma_{\alpha}$ as $w$ tends to a boundary point $\alpha$. This fact could also be deduced from the works of Shapiro [S1] and Cima and Matheson [CM]. Our proof is based on the well-known identity

$$
1-\left|\psi_{w}(z)\right|^{2}=\frac{\left(1-|w|^{2}\right)\left(1-|z|^{2}\right)}{|1-\bar{w} z|^{2}}
$$

Lemma 5.1. For every $\alpha \in \partial \mathbb{D}, \limsup _{w \rightarrow \alpha} \frac{\bar{N}(w)}{-\log |w|} \leq\left\|\sigma_{\alpha}\right\|$.

Proof. Recall from Section 3 that

$$
\bar{N}(w)=\int_{\partial \mathbb{D}} \log \left|\phi_{w}^{*}(\zeta)\right| d m(\zeta)-\log \left|\phi_{w}(0)\right|,
$$

where $\phi_{w}=\psi_{w} \circ \phi$. Since for all $z \in \overline{\mathbb{D}}$,

$$
\lim _{w \rightarrow \alpha} \frac{\log \left|\psi_{w}(z)\right|}{\log |w|}=\lim _{w \rightarrow \alpha} \frac{1-\left|\psi_{w}(z)\right|^{2}}{1-|w|^{2}}=\lim _{w \rightarrow \alpha} \frac{1-|z|^{2}}{|1-\bar{w} z|^{2}}=\frac{1-|z|^{2}}{|\alpha-z|^{2}},
$$

we have by Fatou's lemma that

$$
\limsup _{w \rightarrow \alpha} \frac{\bar{N}(w)}{-\log |w|} \leq-\int_{\partial \mathbb{D}} \frac{1-\left|\phi^{*}(\zeta)\right|^{2}}{\left|\alpha-\phi^{*}(\zeta)\right|^{2}} d m(\zeta)+\frac{1-|\phi(0)|^{2}}{|\alpha-\phi(0)|^{2}}
$$

The right-hand side here is equal to $\left\|\sigma_{\alpha}\right\|$ by fact (c) of Section 2 ,

The essential part of our work concentrates on determining restrictions on $w$ under which the desired limit $\left\|\sigma_{\alpha}\right\|$ is attained, that is, under which we have

$$
\lim _{w \rightarrow \alpha} \frac{N(w)}{-\log |w|}=\left\|\sigma_{\alpha}\right\| \text {. }
$$

It should be stressed that without any such restrictions (5.2) may fail even in the simplest cases: for instance, if $\phi(z)=(1-c)+c z$ for some $0<c<1$, then $N(w)=0$ for all $w \in \mathbb{D} \backslash B(1-c, c)$ but $\left\|\sigma_{1}\right\|=1 / c>0$. In Section 8 more sophisticated examples are used to show that the non-tangential version of this does not hold either.

Before examining the general problem in detail, let us however note that in some special cases it is relatively easy to obtain results of the desired type. One such situation arises when $\sigma_{\alpha}$ is a discrete measure (i.e. consists of point masses only); see Section 10, Another case of special interest is that of inner functions as observed in the corollary below.

Corollary 5.2. If $\phi$ is inner, then $\frac{N(w)}{-\log |w|} \rightarrow\left\|\sigma_{\alpha}\right\|$ as $w \rightarrow \alpha$ in $\mathbb{D} \backslash E$, where $E$ is the polar set of all points $w$ with $N(w)<\bar{N}(w)$.

Proof. Note that $\bar{N}(w)=-\log \left|\phi_{w}(0)\right|$ and apply the idea of the preceding proof.

Returning to the general case, we will next show that (5.2) always holds as $w$ tends to $\alpha$ along some non-tangential sequence. The density condition placed on the sequence will be crucial to our main argument in the next section. 
Proposition 5.3. Let $\Gamma=\Gamma(\alpha, \rho)$ be an arbitrary non-tangential approach region at a point $\alpha \in \partial \mathbb{D}$. Then there exists a sequence $\left(a_{n}\right)$ in $\Gamma$ converging to $\alpha$ such that

$$
\lim _{n \rightarrow \infty} \frac{N\left(a_{n}\right)}{-\log \left|a_{n}\right|}=\left\|\sigma_{\alpha}\right\| .
$$

Moreover, the points $a_{n}$ can be chosen to satisfy the density condition

$$
2^{-n}<1-\left|a_{n}\right|<2^{-n+1} .
$$

The proof of this proposition will depend on a couple of facts from the theory of composition operators. Firstly we record the change of variables formula

$$
\|f \circ \phi\|_{H^{2}}^{2}=|f(\phi(0))|^{2}+2 \int_{\mathbb{D}}\left|f^{\prime}\right|^{2} N d A,
$$

which is valid for any $f$ in the Hardy space $H^{2}$ and where $A$ denotes Lebesgue area measure on $\mathbb{D}$, normalized to have unit total mass. This result can be derived from a general formula for integral means due to C. S. Stanton [St, and it played a fundamental role in Shapiro's S1 solution to the compactness problem for composition operators on $H^{2}$. Two special cases of (5.4) are worth noting. Taking $f(z)=z$ shows that the Nevanlinna counting function is integrable with respect to the area measure: $\int_{\mathbb{D}} N d A \leq \frac{1}{2}$, and taking $\phi(z)=z$ produces the classical Littlewood-Paley identity

$$
\|f\|_{H^{2}}^{2}=|f(0)|^{2}+2 \int_{\mathbb{D}}\left|f^{\prime}\right|^{2} d \lambda
$$

where $d \lambda(z)=-\log |z| d A(z)$.

Secondly we refer to the paper by Cima and Matheson [CM], which presents an alternative solution to the compactness problem in terms of the Aleksandrov measures. The main ingredient of the paper is the identity

$$
\left\|\sigma_{\alpha}\right\|=\lim _{r \rightarrow 1-}\left\|f_{r \alpha} \circ \phi\right\|_{H^{2}}^{2}
$$

involving the normalized reproducing kernel functions $f_{a}(z)=\left(1-|a|^{2}\right)^{1 / 2} /(1-\bar{a} z)$. A simple computation with power series shows that $\left\|f_{a}\right\|_{H^{2}}=1$.

The roots of Proposition [5.3 lie in combining formula (5.4) and equation (5.6), which provide us with the first explicit connection between the Nevanlinna counting function $N$ and measures $\sigma_{\alpha}$. A very implicit form of this connection is already present in Sarason's work Sa1, especially in conjunction with the paper by Shapiro and Sundberg [SS]. We proceed to the proof of the proposition via a lemma.

Lemma 5.4. There exists a constant $c \in] 0, \frac{1}{2}[$, depending on $\rho$ only, such that

$$
\int_{\Gamma(\alpha, \rho, r)}\left|f_{r \alpha}^{\prime}\right|^{2} d \lambda \geq c \quad \text { for } \frac{1}{2}<r<1,
$$

where $\Gamma(\alpha, \rho, r)$ is the set of all $z \in \Gamma(\alpha, \rho)$ with $1-r<1-|z|<2(1-r)$.

Proof. Take $\alpha=1$ for simplicity and write $\Gamma_{r}=\Gamma(1, \rho, r)$. We need to estimate

$$
f_{r}^{\prime}(z)=\frac{r \sqrt{1-r^{2}}}{(1-r z)^{2}} .
$$


It is geometrically obvious that if $z \in \Gamma(1, \rho)$, then $|1-z| \leq c_{1}(1-|z|)$ for some constant $c_{1}>0$ depending on $\rho$ only. Thus, for all $z \in \Gamma_{r}$,

$$
\begin{aligned}
|1-r z| & \leq|1-r|+|r-r z| \leq(1-r)+|1-z| \\
& \leq(1-r)+c_{1}(1-|z|) \leq\left(1+2 c_{1}\right)(1-r) .
\end{aligned}
$$

Since $-\log |z| \geq 1-r$ for all $z \in \Gamma_{r}$, it follows that

$$
\int_{\Gamma_{r}}\left|f_{r}^{\prime}\right|^{2} d \lambda \geq \frac{r^{2}\left(1-r^{2}\right)(1-r)}{\left(1+2 c_{1}\right)^{4}(1-r)^{4}} A\left(\Gamma_{r}\right)=\frac{r^{2}(1+r)}{\left(1+2 c_{1}\right)^{4}} \cdot \frac{A\left(\Gamma_{r}\right)}{(1-r)^{2}} .
$$

The lemma follows because the quotient $A\left(\Gamma_{r}\right) /(1-r)^{2}$ is bounded below by a positive constant depending on $\rho$ only.

Proof of Proposition [5.3. We again take $\alpha=1$. It is easy to see that, as $r \rightarrow 1-$, the derivatives $f_{r}^{\prime}$ tend to zero uniformly outside any neighbourhood of the point 1. Thus we may conclude from (5.4) and (5.6) that

$$
\left\|\sigma_{1}\right\|=\lim _{r \rightarrow 1-} 2 \int_{B_{R}}\left|f_{r}^{\prime}\right|^{2} N d A
$$

where $B_{R}=\mathbb{D} \cap B(1, R)$ for any prescribed $\left.R \in\right] 0,1\left[\right.$. Let $\Gamma_{n}$ be the set of all points $z \in \Gamma$ for which $2^{-n}<1-|z|<2^{-n+1}$; in the notation of the above lemma this is the set $\Gamma\left(1, \rho, r_{n}\right)$ with $r_{n}=1-2^{-n}$. We have to find points $a_{n} \in \Gamma_{n}$ such that (5.3) holds.

Assume, to reach a contradiction, that no such sequence exists. In view of Lemma 5.1 this implies that there are a number $\epsilon>0$ and positive integers $n_{1}<$ $n_{2}<\cdots$ such that

$$
\frac{N(z)}{-\log |z|} \leq\left\|\sigma_{1}\right\|-\epsilon \quad \text { for } z \in \Gamma_{n_{k}} .
$$

Choose a constant $c$ as in Lemma 5.4 and $\delta>0$ so small that $(1-2 c) \delta \leq c \epsilon$. Next use Lemma 5.1 to find a radius $R \in] 0,1[$ such that

$$
\frac{N(z)}{-\log |z|} \leq\left\|\sigma_{1}\right\|+\delta \quad \text { for } z \in B_{R}
$$

For large $k$ we have $\Gamma_{n_{k}} \subset B_{R}$ and then the above inequalities combine to yield

$$
\int_{B_{R}}\left|f_{r_{n_{k}}}^{\prime}\right|^{2} N d A \leq\left(\left\|\sigma_{1}\right\|+\delta\right) \int_{B_{R} \backslash \Gamma_{n_{k}}}\left|f_{r_{n_{k}}}^{\prime}\right|^{2} d \lambda+\left(\left\|\sigma_{1}\right\|-\epsilon\right) \int_{\Gamma_{n_{k}}}\left|f_{r_{n_{k}}}^{\prime}\right|^{2} d \lambda .
$$

But by the Littlewood-Paley identity (5.5) we always have $2 \int\left|f_{r}^{\prime}\right|^{2} d \lambda \leq\left\|f_{r}\right\|_{H^{2}}^{2}=$ 1 , so, by the choices of $c$ and $\delta$, we arrive at the inequality

$$
2 \int_{B_{R}}\left|f_{r_{n_{k}}}^{\prime}\right|^{2} N d A \leq\left\|\sigma_{1}\right\|-c \epsilon .
$$

As $k \rightarrow \infty$, this leads to a contradiction with (5.7), and the proposition is proved.

\section{Capacity estimates}

The purpose of this section is to prove the following proposition, which is a specialized version of Theorem 1.1 and contains the essence of our work. 
Proposition 6.1. Let $\Gamma=\Gamma(\alpha, \rho)$ be an arbitrary non-tangential approach region at a point $\alpha \in \partial \mathbb{D}$. Then there exists a set $E \subset \Gamma$ of finite Green capacity such that

$$
\frac{N(w)}{-\log |w|} \rightarrow\left\|\sigma_{\alpha}\right\| \quad \text { as } w \rightarrow \alpha \text { in } \Gamma \backslash E .
$$

The intuitive idea behind this proposition can be sketched as follows: Note that the function $u(z)=-\left\|\sigma_{\alpha}\right\| \log |z|-N(z)$ is roughly superharmonic and positive near $\alpha$. In addition, thanks to Proposition [5.3, it assumes small values at the points of a sequence that is not too sparse. Therefore $u$ must remain small in a large set. Although this reasoning is not quite precise, a suitable modification of it will work.

We begin with some preliminary observations on Green potentials and capacity. First of all, we recall that if $u$ is a positive superharmonic function on a plane region $\Omega$, then $u$ admits the Riesz decomposition $u=h+v$, where $h$ is the greatest harmonic minorant of $u$ and $v$ is a Green potential, i.e.

$$
v(z)=\int g_{\Omega}(z, w) d \mu(w) .
$$

Here $g_{\Omega}$ is the Green function for $\Omega$ and $\mu$ is a positive Borel measure on $\Omega$, obtained from $u$ by the formula $\Delta u=-2 \pi \mu$ (in the sense of distributions).

By the aid of Riesz decompositions we can use any superharmonic function to estimate the Green capacity as in the next lemma. As before, $D(a, r)$ denotes the pseudo-hyperbolic disc in $\mathbb{D}$ with centre $a$ and radius $r$.

Lemma 6.2. Let $a \in \mathbb{D}, 0<r<R<1$, and assume that $u$ is a positive superharmonic function on $D(a, R)$ with $\Delta u=-2 \pi \mu$. Then there exists a positive constant $c$, depending only on $r$ and $R$, such that

$$
C(\{z \in \bar{D}(a, r): u(z) \geq \delta\}) \leq \frac{\mu(D(a, R))}{\delta-c u(a)}
$$

whenever $\delta>c u(a)$.

Proof. As explained above, we have $u=h+v$, where $h$ is the greatest harmonic minorant of $u$ and

$$
v(z)=\int_{D(a, R)} g_{D(a, R)}(z, w) d \mu(w) .
$$

Since $h$ is positive, Harnack's inequality implies that there is a constant $c$, depending only on $r$ and $R$, such that $h(z) \leq \operatorname{ch}(a)$ for all $z \in \bar{D}(a, r)$. (By considering $u \circ \psi_{a}$ instead of $u$ we see that $c$ does not depend on $a$.) Thus $v(z) \geq u(z)-c u(a)$ for $z \in \bar{D}(a, r)$. Now note that if $g$ is the Green function for $\mathbb{D}$, then $g_{D(a, R)} \leq g$ and consequently $v \leq G_{\mu}$. Hence it follows that whenever $z \in \bar{D}(a, r)$ with $u(z) \geq$ $\delta$, one has $G_{\mu}(z) \geq \delta-c u(a)$. The proof is now finished by an application of Lemma 4.1

The next lemma contains a lower bound for the rate of decay of Green potentials at the boundary of the unit disc.

Lemma 6.3. Let $\mu$ be a positive Borel measure on $\mathbb{D}, a \in \mathbb{D}$ and $0<r<1$. Then

$$
\liminf _{z \rightarrow \alpha} \frac{G_{\mu}(z)}{-\log |z|} \geq \frac{1-|a|^{2}}{|\alpha-a|^{2}} \cdot \frac{1-r}{1+r} \mu(D(a, r))
$$

for all $\alpha \in \partial \mathbb{D}$. 
Proof. If $w \in D(a, r)$, standard inequalities for the pseudo-hyperbolic metric (see e.g. [G] Lemma I.1.4]) yield

$$
\rho(z, w) \leq \frac{\rho(z, a)+\rho(a, w)}{1+\rho(z, a) \rho(a, w)} \leq \frac{r+\rho(z, a)}{1+r \rho(z, a)} .
$$

Assume for simplicity that $\mu(D(a, r))=1$. By the above inequality and the fact that $-\log x \geq \frac{1}{2}\left(1-x^{2}\right)$ we get

$$
G_{\mu}(z)=-\int_{\mathbb{D}} \log \rho(z, w) d \mu(w) \geq \frac{1}{2}\left[1-\left(\frac{r+\rho(z, a)}{1+r \rho(z, a)}\right)^{2}\right],
$$

and after two applications of (5.1),

$$
G_{\mu}(z) \geq \frac{1}{2} \cdot \frac{\left(1-r^{2}\right)\left(1-|a|^{2}\right)\left(1-|z|^{2}\right)}{(1+r \rho(z, a))^{2}|1-\bar{a} z|^{2}} .
$$

The lemma follows because $\left(1-|z|^{2}\right) /(-\log |z|) \rightarrow 2$ and $\rho(z, a) \rightarrow 1$ as $z \rightarrow \alpha$.

We are now ready to carry out the proof of Proposition 6.1.

Proof of Proposition 6.1. Let $\left(a_{n}\right)$ be a sequence in $\Gamma$ as given by Proposition [5.3, It is not difficult to see that whenever $r \in] 0,1[$ is large enough, the pseudo-hyperbolic $\operatorname{discs} \bar{D}\left(a_{n}, r\right)(n=1,2, \ldots)$ cover all points of $\Gamma$ whose modulus is close to 1 . (To be specific, one may show that the pseudo-hyperbolic diameters of the sets $\Gamma_{n}$ used in the proof of Proposition 5.3 are bounded by a constant less than 1 , so it is possible to arrange the $n$ :th disc to cover all of $\Gamma_{n}$.) We first choose such an $r$, then fix some $R \in] r, 1\left[\right.$, and put $D_{n}=\bar{D}\left(a_{n}, r\right), D_{n}^{\prime}=D\left(a_{n}, R\right)$ and

$$
A_{n}^{\epsilon}=\left\{z \in D_{n}: \frac{\bar{N}(z)}{-\log |z|} \leq\left\|\sigma_{\alpha}\right\|-\epsilon\right\},
$$

where $\epsilon>0$ is fixed but arbitrary. In view of Lemma 5.1, the fact that $N=\bar{N}$ q.e. and the countable subadditivity of the Green capacity, it is now sufficient to prove that $\sum_{n=1}^{\infty} C\left(A_{n}^{\epsilon}\right)<\infty$. In fact, we can then choose integers $n_{k}$ such that $\sum_{n=n_{k}}^{\infty} C\left(A_{n}^{1 / k}\right)<1 / k^{2}$ for each $k$ and put $E=\Gamma \cap\left(\bigcup_{k=1}^{\infty} \bigcup_{n=n_{k}}^{\infty} A_{n}^{1 / k}\right)$.

We fix some small $\delta>0$ (the actual choice will become evident in a moment). Using Proposition 5.3 and Lemma [5.1] we see that for large $n$, say $n \geq n_{0}$, the following are satisfied:

$$
\begin{gathered}
\left|a_{n}\right| \geq 1 / 2 \quad \text { and } \quad D_{n}^{\prime} \cap\{0, \phi(0)\}=\emptyset, \\
\frac{\bar{N}\left(a_{n}\right)}{-\log \left|a_{n}\right|} \geq\left\|\sigma_{\alpha}\right\|-\delta, \\
\frac{\bar{N}(z)}{-\log |z|} \leq\left\|\sigma_{\alpha}\right\|+\delta \text { on } D_{n}^{\prime} .
\end{gathered}
$$

We also require the following fact about pseudo-hyperbolic discs: for every $s \in] 0,1[$ there are positive constants $\gamma_{s}$ and $\gamma_{s}^{\prime}$ such that

$$
\gamma_{s}(1-|a|) \leq 1-|z| \leq \gamma_{s}^{\prime}(1-|a|) \quad \text { for } z \in \bar{D}(a, s) .
$$

(In fact, $\gamma_{s}=1 / \gamma_{s}^{\prime}=(1-s) /(1+s)$ will do.)

Now consider the function $u(z)=-\bar{N}(z)-\left(\left\|\sigma_{\alpha}\right\|+\delta\right) \log |z|$. It is superharmonic in $\mathbb{D} \backslash\{0, \phi(0)\}$ with $\Delta u=-\Delta \bar{N}=-2 \pi \mu$ there, $\mu$ being the distribution of 
the boundary values of $\phi$ (cf. Section 3). In addition, it follows from the above inequalities that if $n \geq n_{0}$, then $u$ is positive on $D_{n}^{\prime}$ with

$$
u\left(a_{n}\right) \leq-2 \delta \log \left|a_{n}\right| \leq 4 \delta\left(1-\left|a_{n}\right|\right),
$$

and, for $z \in A_{n}^{\epsilon}$,

$$
u(z) \geq-(\epsilon+\delta) \log |z| \geq(\epsilon+\delta) \gamma_{r}\left(1-\left|a_{n}\right|\right) .
$$

Hence an application of Lemma 6.2 yields

$$
C\left(A_{n}^{\epsilon}\right) \leq \frac{\mu\left(D_{n}^{\prime}\right)}{(\epsilon+\delta) \gamma_{r}\left(1-\left|a_{n}\right|\right)-c \cdot 4 \delta\left(1-\left|a_{n}\right|\right)}=c_{1} \frac{\mu\left(D_{n}^{\prime}\right)}{1-\left|a_{n}\right|},
$$

where $c_{1}>0$ is independent of $n$. Note here that the number $\delta$ must be sufficiently small in order that the denominator be positive.

We will complete the proof by showing that

$$
\sum_{n=1}^{\infty} \frac{\mu\left(D_{n}^{\prime}\right)}{1-\left|a_{n}\right|}<\infty
$$

We argue by contradiction. Observe that due to the density condition satisfied by the points $a_{n}$ and inequalities (6.1) there exists a positive integer $j$ such that any two discs $D_{n}^{\prime}$ and $D_{n+l}^{\prime}$ are disjoint if $l \geq j$. Therefore, if (6.2) fails, it is possible to extract indices $n_{1}<n_{2}<\cdots$ (of the form $n_{k+1}=n_{k}+j$ ) for which the discs $D_{n_{k}}^{\prime}$ are disjoint and satisfy

$$
\sum_{k=1}^{\infty} \frac{\mu\left(D_{n_{k}}^{\prime}\right)}{1-\left|a_{n_{k}}\right|}=\infty
$$

Let $\mu_{k}$ be the restriction of $\mu$ to $D_{n_{k}}^{\prime}$. By Lemma 6.3 we have

$$
\liminf _{z \rightarrow \alpha} \frac{G_{\mu_{k}}(z)}{-\log |z|} \geq \frac{1-\left|a_{n_{k}}\right|^{2}}{\left|\alpha-a_{n_{k}}\right|^{2}} \cdot \frac{1-R}{1+R} \mu\left(D_{n_{k}}^{\prime}\right) .
$$

Since the points $a_{n_{k}}$ all lie in the non-tangential approach region $\Gamma$, we have the inequality $c_{2}\left|\alpha-a_{n_{k}}\right| \leq\left(1-\left|a_{n_{k}}\right|\right)$ for some constant $c_{2}>0$, and consequently

$$
\liminf _{z \rightarrow \alpha} \frac{G_{\mu_{k}}(z)}{-\log |z|} \geq c_{2}^{2} \frac{1-R}{1+R} \frac{\mu\left(D_{n_{k}}^{\prime}\right)}{1-\left|a_{n_{k}}\right|} .
$$

Summing over $k$ and noting that $G_{\mu} \geq \sum_{k=1}^{\infty} G_{\mu_{k}}$ we get that $G_{\mu}(z) /(-\log |z|) \rightarrow$ $\infty$ as $z \rightarrow \alpha$. This is however impossible since $\bar{N}(z)=-G_{\mu}(z)-\log \left|\phi_{z}(0)\right|$ is always non-negative. Therefore (6.2) holds and the proof is complete.

\section{Completion of the proof}

In this section we finish the proof of Theorem 1.1 by showing that the assertion about the convergence of measures $M_{w}$ to $\sigma_{\alpha}$ in the weak* sense reduces to establishing the convergence of the total mass $\left\|M_{w}\right\|$ to $\left\|\sigma_{\alpha}\right\|$, which was carried out in the preceding section. Indeed, the theorem follows immediately on combining the following proposition with Proposition 6.1.

Proposition 7.1. Let $\alpha \in \partial \mathbb{D}$ and assume that $\left(w_{n}\right)$ is a sequence in $\mathbb{D}$ converging to $\alpha$ such that $\frac{N\left(w_{n}\right)}{-\log \left|w_{n}\right|} \rightarrow\left\|\sigma_{\alpha}\right\|$. Then $M_{w_{n}} \rightarrow \sigma_{\alpha}$ weak* 
The proof of this proposition consists of a simple change of variables trick utilizing the disc automorphisms $\psi_{a}(z)=(a-z) /(1-\bar{a} z)$ for $a \in \mathbb{D}$. Note that

$$
\psi_{a}^{\prime}(z)=\frac{|a|^{2}-1}{(1-\bar{a} z)^{2}} .
$$

In the next lemma, we let $\tau_{\alpha, a}$ denote the Aleksandrov measure of the composition $\phi \circ \psi_{a}$ at the point $\alpha \in \partial \mathbb{D}$ and $\sigma_{\alpha, a}$ is its singular part.

Lemma 7.2. The measures $\tau_{\alpha, a}$ satisfy

$$
d \tau_{\alpha, a}(\zeta)=\frac{|1-\bar{a} \zeta|^{2}}{1-|a|^{2}} d\left(\tau_{\alpha} \circ \psi_{a}\right)(\zeta)
$$

where $\tau_{\alpha} \circ \psi_{a}$ is the pull-back of $\tau_{\alpha}$ under $\psi_{a}$, i.e. $\left(\tau_{\alpha} \circ \psi_{a}\right)(E)=\tau_{\alpha}\left(\psi_{a}(E)\right)$ for all Borel sets $E \subset \partial \mathbb{D}$. The corresponding result holds for the singular parts $\sigma_{\alpha, a}$ as well, and therefore

$$
\left\|\sigma_{\alpha, a}\right\|=\int_{\partial \mathbb{D}} \frac{1-|a|^{2}}{|1-\bar{a} \zeta|^{2}} d \sigma_{\alpha}(\zeta)
$$

Proof. Fix a point $\zeta \in \partial \mathbb{D}$ for the moment. Then we have the identity

$$
\frac{1-\left|\psi_{a}(z)\right|^{2}}{\left|\zeta-\psi_{a}(z)\right|^{2}}=\frac{1-|z|^{2}}{\left|\psi_{a}(\zeta)-z\right|^{2}}\left|\psi_{a}^{\prime}(\zeta)\right|
$$

as can be verified by direct computation or using fact (e) of Section 2 and noting that $\psi_{a}$ assumes the boundary limit $\zeta$ precisely at the point $\xi=\psi_{a}(\zeta)$, where $\psi_{a}^{\prime}(\xi)=\psi_{a}^{\prime}(\zeta)^{-1}$. Integrating with respect to $\tau_{\alpha}$, the Aleksandrov measure of $\phi$ at the point $\alpha$, we obtain

$$
\begin{aligned}
\frac{1-\left|\phi\left(\psi_{a}(z)\right)\right|^{2}}{\left|\alpha-\phi\left(\psi_{a}(z)\right)\right|^{2}} & =\int_{\partial \mathbb{D}} \frac{1-|z|^{2}}{\left|\psi_{a}(\zeta)-z\right|^{2}}\left|\psi_{a}^{\prime}(\zeta)\right| d \tau_{\alpha}(\zeta) \\
& =\int_{\partial \mathbb{D}} \frac{1-|z|^{2}}{|\zeta-z|^{2}}\left|\psi_{a}^{\prime}(\zeta)\right|^{-1} d\left(\tau_{\alpha} \circ \psi_{a}\right)(\zeta) .
\end{aligned}
$$

The desired identity for $\tau_{\alpha, a}$ follows immediately. The last statements are obtained by identifying the singular parts here (since $\psi_{a}$ is absolutely continuous on the circle) and performing a change of variables in integration.

We will next derive a similar result for the Nevanlinna counting measures. To this end, it is useful to introduce the following modifications of them:

$$
\widetilde{M}_{w}=\sum_{z \in \phi^{-1}\{w\}} \frac{1-|z|^{2}}{1-|w|^{2}} \delta_{z}
$$

These measures are asymptotically equivalent to the ordinary Nevanlinna counting measures in the sense that $\left\|M_{w}-\widetilde{M}_{w}\right\| \rightarrow 0$ as $|w| \rightarrow 1-$. Indeed,

$$
\begin{aligned}
\left\|M_{w}-\widetilde{M}_{w}\right\| & =\sum_{z \in \phi^{-1}\{w\}}\left|\frac{-\log |z|}{-\log |w|}-\frac{1-|z|^{2}}{1-|w|^{2}}\right| \\
& \leq \frac{N(w)}{-\log |w|} \cdot \sup _{z \in \phi^{-1}\{w\}}\left|1-\frac{-\log |w|}{1-|w|^{2}} \cdot \frac{1-|z|^{2}}{-\log |z|}\right|,
\end{aligned}
$$

where the quotient $N(w) /(-\log |w|)$ stays bounded (by Littlewood's inequality) and the supremum tends to zero (because, by the Schwarz lemma, the moduli of the pre-images of $w$ tend to 1 uniformly) as $|w| \rightarrow 1-$. 
By analogy with the notation used in Lemma 7.2 let $\widetilde{M}_{w, a}$ denote the modified counting measures (7.1) defined in terms of $\phi \circ \psi_{a}$ instead of $\phi$.

Lemma 7.3. The measures $\widetilde{M}_{w, a}$ satisfy

$$
\left\|\widetilde{M}_{w, a}\right\|=\int_{\mathbb{D}} \frac{1-|a|^{2}}{|1-\bar{a} z|^{2}} d \widetilde{M}_{w}(z) .
$$

Proof. By the self-inverse property of $\psi_{a}$ and equation (5.1),

$$
\left\|\widetilde{M}_{w, a}\right\|=\sum_{z \in \phi^{-1}\{w\}} \frac{1-\left|\psi_{a}(z)\right|^{2}}{1-|w|^{2}}=\sum_{z \in \phi^{-1}\{w\}} \frac{1-|a|^{2}}{|1-\bar{a} z|^{2}} \cdot \frac{1-|z|^{2}}{1-|w|^{2}} .
$$

Comparing this with the definition of $\widetilde{M}_{w}$, we get what we want.

After these preliminaries we can prove Proposition 7.1 (and, as noted before, finish the proof of Theorem 1.1).

Proof of Proposition 7.1. Let $\left(w_{n}\right)$ be a sequence as in the assumption. If $a \in \mathbb{D}$ is given, we may apply Lemma 5.1 to the function $\phi \circ \psi_{a}$ with the result that

$$
\limsup _{n \rightarrow \infty} \frac{N_{\phi \circ \psi_{a}}\left(w_{n}\right)}{-\log \left|w_{n}\right|} \leq\left\|\sigma_{\alpha, a}\right\|
$$

According to Lemmas 7.2 and 7.3 plus the asymptotic equivalence of $M$ and $\widetilde{M}$, this yields

$$
\limsup _{n \rightarrow \infty} \int_{\mathbb{D}} \frac{1-|a|^{2}}{|1-\bar{a} z|^{2}} d M_{w_{n}}(z) \leq \int_{\partial \mathbb{D}} \frac{1-|a|^{2}}{|1-\bar{a} \zeta|^{2}} d \sigma_{\alpha}(\zeta) .
$$

Now let $\mu$ be any weak* limit point of the sequence $\left(M_{w_{n}}\right)$. Then the support of $\mu$ is contained in $\partial \mathbb{D}$, and the inequality above implies that the Poisson integral of $\mu$ is majorized by that of $\sigma_{\alpha}$ at every point $a \in \mathbb{D}$. Therefore the difference $\sigma_{\alpha}-\mu$ is a positive measure supported on $\partial \mathbb{D}$. By assumption, however, $\left\|\sigma_{\alpha}\right\|=\|\mu\|$, and consequently $\sigma_{\alpha}=\mu$. Thus $\sigma_{\alpha}$ is the only possible weak ${ }^{*}$ limit point of the sequence $\left(M_{w_{n}}\right)$. By weak* compactness we conclude that $M_{w_{n}} \rightarrow \sigma_{\alpha}$ in the weak* sense.

\section{OPTIMALITY AND OTHER RESULTS}

\section{Sharpness of TheOrem 1.1}

In this section we demonstrate that the capacity condition stated in Theorem 1.1 is optimal. Recall for this purpose that for a set $E \subset \Gamma(1, \rho)$ to be of finite Green capacity, it is necessary and sufficient that the capacity of $E \cap B(1, r)$ decays to zero as $r \rightarrow 0+$. (The verification of this is an exercise in using the asymptotic additivity property of Green capacity discussed at the end of Section 4) The following proposition shows that it is not possible to give an upper bound for the corresponding rate of decay for the exceptional set in Theorem 1.1 .

Proposition 8.1. Let $\Gamma=\Gamma(1, \rho)$ be an arbitrary non-tangential approach region at the point 1 , and let $\left(t_{n}\right)$ and $\left(r_{n}\right)$ be two sequences of positive reals decreasing strictly to zero. There exists a holomorphic function $\phi: \mathbb{D} \rightarrow \mathbb{D}$ such that

(1) $\sigma_{1}$, the singular part of the Aleksandrov measure of $\phi$ at the point 1 , is non-vanishing; 
(2) for large $n$ we have $C\left(\left\{w \in \Gamma \cap B\left(1, r_{n}\right): M_{w}=0\right\}\right)>t_{n}$, where $M_{w}$ is the Nevanlinna counting measure of $\phi$ at $w$.

Our construction makes use of the following setup. We let $\Omega$ be the region obtained from the unit disc by removing a sequence of closed pseudo-hyperbolic $\operatorname{discs} D_{n}=\bar{D}\left(a_{n}, s_{n}\right)$ where $0<s_{n}<\frac{1}{2}<a_{n}<1$ for each $n \geq 1$. We will also make the sequence $\left(a_{n}\right)$ increase to 1 so rapidly that the discs $D_{n}$ are disjoint. We then let $\phi$ be an analytic covering map from $\mathbb{D}$ onto $\Omega$ such that $\phi(0)=0$. Now $M_{w}=0$ for all $w$ in the set $\bigcup_{n=1}^{\infty} D_{n}$, and our task is to specify additional conditions on the centres $a_{n}$ and radii $s_{n}$ in such a way that the conclusions of the above proposition hold.

Since $\phi$ is bounded and holomorphic, it has non-tangential limits $\phi^{*}(\zeta)$ almost everywhere on $\partial \mathbb{D}$, and a well-known property of covering maps is that these all lie in $\partial \Omega$. We will now acquire some information on the distribution of these boundary values. The proof of the following lemma is found in [F, Prop. 5.2.5].

Lemma 8.2. Let $\Omega \subset \mathbb{D}$ be a region and $\phi: \mathbb{D} \rightarrow \Omega$ an analytic covering map. If $A \subset \mathbb{D} \cap \partial \Omega$ is a Borel set of Green capacity zero, then the set of all points $\zeta \in \partial \mathbb{D}$ with $\phi^{*}(\zeta) \in A$ has Lebesgue measure zero.

Lemma 8.3. Let $\Omega \subset \mathbb{D}$ be a region containing the origin and $\phi: \mathbb{D} \rightarrow \Omega$ an analytic covering map with $\phi(0)=0$. If $K \subset \mathbb{D} \cap \partial \Omega$ is compact and $S=\{\zeta \in \partial \mathbb{D}$ : $\left.\phi^{*}(\zeta) \in K\right\}$, then

$$
m(S) \leq G_{\mu_{0}}(0)=-\int \log |z| d \mu_{0}(z)
$$

where $\mu_{0}$ is the capacitary distribution of $K$.

Proof. Consider the function $u(z)=G_{\mu_{0}}(\phi(z))$. It is harmonic in the unit disc with values between zero and one. Furthermore, since $G_{\mu_{0}}(z)=1$ for q.e. $z \in K$, it follows from the lower semicontinuity of $G_{\mu_{0}}$ and the preceding lemma that the boundary limits $u^{*}(\zeta)$ are equal to 1 for a.e. $\zeta \in S$. Hence

$$
m(S) \leq \int_{\partial \mathbb{D}} u^{*}(\zeta) d m(\zeta)=u(0)=G_{\mu_{0}}(0) .
$$

We now return to the setup described before the lemmas. Our first goal is to seek conditions under which (1) holds true for the covering map $\phi$. By fact (c) of Section 2] we have

$$
\left\|\sigma_{1}\right\|=1-\sum_{n=1}^{\infty} \int_{S_{n}} \frac{1-\left|\phi^{*}(\zeta)\right|^{2}}{\left|1-\phi^{*}(\zeta)\right|^{2}} d m(\zeta)
$$

where $S_{n} \subset \partial \mathbb{D}$ consists of those $\zeta$ for which $\phi^{*}(\zeta) \in D_{n}$. To estimate this, we deduce from (6.1) that for $\zeta \in S_{n}$,

$$
\frac{1-\left|\phi^{*}(\zeta)\right|^{2}}{\left|1-\phi^{*}(\zeta)\right|^{2}} \leq \frac{2}{1-\left|\phi^{*}(\zeta)\right|} \leq \frac{2}{\gamma\left(1-a_{n}\right)},
$$

where $\gamma>0$ is a constant. In addition, by Lemma 8.3 and equation (4.1) we have

$$
m\left(S_{n}\right) \leq c_{n}\left(-\log a_{n}\right) \leq 2 c_{n}\left(1-a_{n}\right),
$$


where $c_{n}$ is the Green capacity of $D_{n}$. These inequalities then combine to give

$$
\left\|\sigma_{1}\right\| \geq 1-\frac{2}{\gamma} \sum_{n=1}^{\infty} \frac{m\left(S_{n}\right)}{1-a_{n}} \geq 1-\frac{4}{\gamma} \sum_{n=1}^{\infty} c_{n} .
$$

Thus, for condition (1) to hold, it is sufficient to have $\sum_{n=1}^{\infty} c_{n}<\gamma / 4$.

Let us next consider condition (2). Recall from Section 4 that the Green capacity of the disc $D_{n}$ depends on its pseudo-hyperbolic radius through the formula $c_{n}=$ $\left(-\log s_{n}\right)^{-1}$. By discarding a finite number of members from the sequences $\left(t_{n}\right)$ and $\left(r_{n}\right)$, we may assume that the differences $d_{n}=t_{n}-t_{n+1}$ are all less than $(\log 2)^{-1}$ and that their sum is less than $\gamma / 4$. Then we can fix the radii $0<s_{n}<\frac{1}{2}$ so that $c_{n}>d_{n}$ for each $n$ and $\sum_{n=1}^{\infty} c_{n}<\gamma / 4$. Now we proceed to choose the centres $\frac{1}{2}<a_{1}<a_{2}<\cdots<1$ such that the discs $D_{n}=\bar{D}\left(a_{n}, s_{n}\right)$ satisfy $D_{n} \subset B\left(1, r_{n}\right)$. Moreover, we may arrange the sequence $\left(a_{n}\right)$ to increase to 1 so rapidly that for each given $m$, the pseudo-hyperbolic distances between the discs $D_{m}, D_{m+1}, D_{m+2}, \ldots$ are large enough in order that

$$
C\left(\bigcup_{n=m}^{\infty} D_{n}\right)>\sum_{n=m}^{\infty} d_{n}=t_{m}
$$

More precisely, we first fix $a_{1}$ and then choose $\left(a_{n}\right)_{n \geq 2}$ to increase to 1 so rapidly that the "asymptotic" additivity property of the Green capacity (cf. Section 4) forces (8.1) to be true for $m=1$. At the next step we hold $a_{1}$ and $a_{2}$ fixed and, if needed, move $\left(a_{n}\right)_{n \geq 3}$ in order to get (8.1) for $m=2$. It is clear how to continue the inductive procedure, whence we obtain (8.1) for all $\mathrm{m}$. Since $s_{n} \rightarrow 0$, it is easily seen that $D_{n} \subset \Gamma$ for large $n$, and conclusions (1) and (2) of Proposition 8.1 hold.

\section{Proof of Theorem 1.2}

The proof of Theorem 1.2 turns out to be considerably simpler than that of Theorem 1.1. Instead of Proposition 6.1 we will now exploit the following lemma.

Lemma 9.1. For every measurable set $E \subset \partial \mathbb{D}$,

$$
\lim _{r \rightarrow 1-} \int_{E} \frac{N(r \alpha)}{-\log r} d m(\alpha)=\int_{E}\left\|\sigma_{\alpha}\right\| d m(\alpha) .
$$

Proof. First of all note that since $\bar{N}$ differs from $N$ in a polar set only and such sets always have Hausdorff dimension zero, the integral on the left is unchanged if $N$ is replaced by $\bar{N}$. Now it follows from Lemma 5.1 and Fatou's lemma that

$$
\limsup _{r \rightarrow 1-} \int_{E} \frac{\bar{N}(r \alpha)}{-\log r} d m(\alpha) \leq \int_{E}\left\|\sigma_{\alpha}\right\| d m(\alpha)
$$

(recall that $\bar{N}(r \alpha)=O(-\log r)$ by Littlewood's inequality). Since the same result holds for integrals over the complement of $E$, we see that in order to prove the lemma it is enough to treat the special case $E=\partial \mathbb{D}$.

Write $A_{r}=\left\{\zeta \in \partial \mathbb{D}:\left|\phi^{*}(\zeta)\right|<r\right\}$ for $0<r \leq 1$ and $B=\left\{\zeta \in \partial \mathbb{D}:\left|\phi^{*}(\zeta)\right|=1\right\}$. Since, by fact (c) of Section 2

$$
\left\|\sigma_{\alpha}\right\|=\frac{1-|\phi(0)|^{2}}{|\alpha-\phi(0)|^{2}}-\int_{\partial \mathbb{D}} \frac{1-\left|\phi^{*}(\zeta)\right|^{2}}{\left|\alpha-\phi^{*}(\zeta)\right|^{2}} d m(\alpha)
$$


an application of Fubini's theorem shows that

$$
\int_{\partial \mathbb{D}}\left\|\sigma_{\alpha}\right\| d m(\alpha)=1-m\left(A_{1}\right)=m(B) .
$$

Turning to the Nevanlinna counting function, we first deduce from 3.1 that

$$
\bar{N}(r \alpha)=-\log |r \alpha-\phi(0)|+\int_{\partial \mathbb{D}} \log \left|r \alpha-\phi^{*}(\zeta)\right| d m(\zeta) .
$$

According to Fubini's theorem and the formula $\int_{\partial \mathbb{D}} \log |r \alpha-z| d m(\alpha)=\log (r \vee|z|)$ it follows that for all $r>|\phi(0)|$,

$$
\int_{\partial \mathbb{D}} \bar{N}(r \alpha) d m(\alpha)=-\log r+m\left(A_{r}\right) \log r+\int_{A_{1} \backslash A_{r}} \log \left|\phi^{*}(\zeta)\right| d m(\zeta) .
$$

Consequently,

$$
\int_{\partial \mathbb{D}} \frac{\bar{N}(r \alpha)}{-\log r} d m(\alpha)=m\left(\partial \mathbb{D} \backslash A_{r}\right)-\int_{A_{1} \backslash A_{r}} \frac{\log \left|\phi^{*}(\zeta)\right|}{\log r} d m(\zeta) .
$$

As $r \rightarrow 1-$, we have $m\left(\partial \mathbb{D} \backslash A_{r}\right) \rightarrow m(B)$ while the integral on the right tends to zero by the bounded convergence theorem. The desired result follows immediately.

The proof of Theorem 1.2 is now completed by an argument similar to the one used to establish Proposition 7.1. Let a point $a \in \mathbb{D}$ and a measurable set $E \subset \partial \mathbb{D}$ be fixed for the moment. Then we may apply the above lemma to the function $\phi \circ \psi_{a}$ with the result that

$$
\lim _{r \rightarrow 1-} \int_{E} \frac{N_{\phi \circ \psi_{a}}(r \alpha)}{-\log r} d m(\alpha)=\int_{E}\left\|\sigma_{\alpha, a}\right\| d m(\alpha),
$$

where $\sigma_{\alpha, a}$ is the singular part of the Aleksandrov measure of $\phi \circ \psi_{a}$ at $\alpha$. As in the proof of Proposition 7.1 it follows from Lemmas 7.2 and 7.3 that

$$
\lim _{r \rightarrow 1-} \int_{E}\left[\int_{\mathbb{D}} \frac{1-|a|^{2}}{|1-\bar{a} z|^{2}} d M_{r \alpha}(z)\right] d m(\alpha)=\int_{E}\left[\int_{\partial \mathbb{D}} \frac{1-|a|^{2}}{|1-\bar{a} \zeta|^{2}} d \sigma_{\alpha}(\zeta)\right] d m(\alpha) .
$$

This proves Theorem 1.2 for test functions of the form $g(\alpha, z)=\chi_{E}(\alpha) \frac{1-|a|^{2}}{|1-\bar{a} z|^{2}}$. The general result follows by approximation.

\section{Angular Derivatives}

This last section contains some additional remarks which are mostly known to specialists.

As before, we let $\phi$ be a holomorphic self-map of the unit disc and we denote by $\phi^{*}(\zeta)$ its non-tangential boundary limit at a point $\zeta \in \partial \mathbb{D}$ whenever the limit exists. We say that $\phi$ has a finite angular derivative at $\zeta$ if there exists a point $\alpha \in \partial \mathbb{D}$ such that the difference quotient $(\phi(z)-\alpha) /(z-\zeta)$ tends to a finite limit as $z$ tends to $\zeta$ non-tangentially. This limit is then called the angular derivative of $\phi$ at $\zeta$ and denoted by $\phi^{\prime}(\zeta)$. Clearly, in this case $\phi^{*}(\zeta)=\alpha$.

The main result about angular derivatives is the following classical theorem [C] Sec. 298, Theorem 2.1]:

Theorem 10.1 (Julia-Carathéodory). For $\zeta \in \partial \mathbb{D}$, the following are equivalent:

(1) $\phi$ has a finite angular derivative at $\zeta$.

(2) $\phi$ has a non-tangential limit of modulus 1 at $\zeta$, and $\phi^{\prime}$ has a finite nontangential limit at $\zeta$. 
(3) The quantity $d(\zeta)=\liminf _{z \rightarrow \zeta}(1-|\phi(z)|) /(1-|z|)$ is finite.

Furthermore, in this case the non-tangential limit of $\phi^{\prime}$ at $\zeta$, the angular derivative $\phi^{\prime}(\zeta)$ and the number $d(\zeta) \phi^{*}(\zeta) \bar{\zeta}$ all agree, and the limit inferior in (3) is a nontangential limit.

Note that by the Schwarz lemma the quantity $d(\zeta)$ must always be positive. As a consequence, the angular derivative is never zero.

The existence of a finite angular derivative at a point $\zeta \in \partial \mathbb{D}$ guarantees that $\phi$ is "non-tangentially conformal" at $\zeta$ in the sense that it maps any curve in $\mathbb{D}$ terminating at $\zeta$ and making an angle $\theta \in]-\pi / 2, \pi / 2[$ with the radius to $\zeta$ onto a curve terminating at $\alpha=\phi^{*}(\zeta)$ and making the same angle $\theta$ with the corresponding radius. The standard argument used to prove conformality of holomorphic functions at points of non-vanishing derivative shows this. Moreover, one has the following important corollary (see [S1]): whenever $0<\rho<\rho^{\prime}<1$, there exists a radius $r \in] 0,1\left[\right.$ such that the image of the non-tangential region $\Gamma\left(\zeta, \rho^{\prime}\right)$ under $\phi$ contains the set $\Gamma(\alpha, \rho) \backslash B(0, r)$, which we will denote by $\Gamma_{r}(\alpha, \rho)$.

There is a simple connection between angular derivatives and Aleksandrov measures. The proof of the following can be found (somewhat implicitly) in [F pp. 222225] and [G, pp. 43-45]. A different approach is given in [Sa2, VI-7].

Lemma 10.2. For $\alpha \in \partial \mathbb{D}$, the measure $\sigma_{\alpha}$ has an atom at a point $\zeta \in \partial \mathbb{D}$ if and only if $\phi^{*}(\zeta)=\alpha$ and $\phi$ has a finite angular derivative at $\zeta$. In this case $\left|\phi^{\prime}(\zeta)\right|=1 / \sigma_{\alpha}(\{\zeta\})$.

Non-tangential conformality entails a considerable strengthening of Theorem 1.1 in the case when $\sigma_{\alpha}$ is discrete (i.e. consists of point masses only). The following argument appears in [S1, 3.3].

Theorem 10.3. Suppose $\alpha \in \partial \mathbb{D}$ and $\sigma_{\alpha}$ is discrete. Then

$$
M_{w} \rightarrow \sigma_{\alpha} \text { weak }^{*} \quad \text { as } w \rightarrow \alpha \text { non-tangentially. }
$$

Proof. By the hypothesis we have $\sigma_{\alpha}=\sum_{k} a_{k} \delta_{\zeta_{k}}$ where $\left(\zeta_{k}\right)$ is a (finite or infinite) sequence of distinct points in $\partial \mathbb{D}$ and $a_{k}>0$ for each $k$. Moreover, the above lemma implies that $\phi$ has a finite angular derivative at each $\zeta_{k}$ and $\left|\phi^{\prime}\left(\zeta_{k}\right)\right|=1 / a_{k}$. According to Proposition 7.1] it is enough to show that

$$
\frac{N(w)}{-\log |w|} \rightarrow \sum_{k} a_{k} \quad \text { as } w \rightarrow \alpha \text { non-tangentially. }
$$

Let $\Gamma(\alpha, \rho)$ be an arbitrary non-tangential approach region at $\alpha$ and choose a number $\rho^{\prime}$ with $\rho<\rho^{\prime}<1$. Also fix any positive integer $n$, not greater than the number of points $\zeta_{k}$. Since $\phi$ is non-tangentially conformal at points $\zeta_{1}, \ldots, \zeta_{n}$, there exists a radius $r \in] 0,1\left[\right.$ such that $\bigcap_{k=1}^{n} \phi\left(\Gamma\left(\zeta_{k}, \rho^{\prime}\right)\right)$ contains the set $\Gamma=\Gamma_{r}(\alpha, \rho)$. Thus, for each $w \in \Gamma$ we can find points $z_{k}(w) \in \Gamma\left(\zeta_{k}, \rho^{\prime}\right)(k=1, \ldots, n)$ for which $\phi\left(z_{k}(w)\right)=w$. Then

$$
\frac{N(w)}{-\log |w|} \geq \sum_{k=1}^{n} \frac{-\log \left|z_{k}(w)\right|}{-\log |w|}
$$

As $w \rightarrow \alpha$ in $\Gamma$, the Schwarz lemma guarantees that $z_{k}(w) \rightarrow \zeta_{k}$ in $\Gamma\left(\zeta_{k}, \rho^{\prime}\right)$. Hence, by the Julia-Carathéodory theorem,

$$
\lim \frac{-\log \left|z_{k}(w)\right|}{-\log |w|}=\lim \frac{1-\left|z_{k}(w)\right|}{1-|w|}=\frac{1}{\left|\phi^{\prime}\left(\zeta_{k}\right)\right|}=a_{k} .
$$


Since $n$ and $\rho$ were arbitrary, we conclude that

$$
\liminf \frac{N(w)}{-\log |w|} \geq \sum_{k} a_{k} \quad \text { as } w \rightarrow \alpha \text { non-tangentially. }
$$

The desired result (10.1) follows from this and Lemma 5.1 .

Theorem 1.1 clearly implies that all the measures $\sigma_{\alpha}$ are discrete in the case when $\phi$ is boundedly valent. In general, however, it seems quite difficult to characterize those functions $\phi$ (or Aleksandrov measures) for which the exceptional set of Theorem 1.1 disappears as above. In particular, there is no converse to Theorem 10.3 in the sense that the presence of a continuous singularity in $\sigma_{\alpha}$ would imply that $\lim N\left(w_{n}\right) /\left(-\log \left|w_{n}\right|\right)<\left\|\sigma_{\alpha}\right\|$ for some sequence $\left(w_{n}\right)$ converging to $\alpha$. To demonstrate this we borrow an example from $\mathrm{BCP}$.

Example 10.4. Let $C$ be the usual Cantor ternary set regarded as a subset of the unit circle via the mapping $\theta \mapsto e^{i \theta}$. Supported on $C$ there is a natural continuously singular probability measure $\mu$. (Recall that $C$ is the intersection of closed sets $C_{n}$, each of which is made up of $2^{n}$ disjoint arcs of length $3^{-n}$. The measure $\mu$ assigns a mass $2^{-n}$ to each of these arcs.) Define a holomorphic self-map $\phi$ of $\mathbb{D}$ through the Herglotz formula

$$
\frac{1+\phi(z)}{1-\phi(z)}=\int_{\partial \mathbb{D}} \frac{\zeta+z}{\zeta-z} d \mu(\zeta)
$$

so that $\mu$ becomes the Aleksandrov measure of $\phi$ at the point 1:

$$
\frac{1-|\phi(z)|^{2}}{|1-\phi(z)|^{2}}=\int_{\partial \mathbb{D}} P(z, \zeta) d \mu(\zeta)
$$

Obviously, $\phi$ is an inner function fixing the origin. Our goal is now to show that the Frostman transforms of $\phi$ are all Blaschke products and hence $N(w)=-\log |w|$ for all $w \in \mathbb{D}$. Then $N(w) /(-\log |w|)=\|\mu\|$ everywhere, even though $\mu$ is continuous and therefore $\phi$ has no finite angular derivative at any boundary point $\zeta$ for which $\phi^{*}(\zeta)=1$.

Let us first observe, by a trivial computation, that the symmetric derivative of $\mu$ (with respect to the Lebesgue measure) is infinite at all points of $C$. Thus the Poisson integral of $\mu$ has infinity as its non-tangential limit everywhere on $C$. By (10.3) this yields $\phi^{*}(\zeta)=1$ for all $\zeta \in C$. On the other hand, it is easily seen from (10.2) that $\phi$ extends holomorphically across the open set $\partial \mathbb{D} \backslash C$, necessarily being unimodular there. Hence $\phi$ has unimodular boundary values at all points of the unit circle, and the same is consequently true of all the Frostman transforms $\psi_{w} \circ \phi$. Because every singular inner function has non-tangential limit 0 at some point of $\partial \mathbb{D}$, it follows that the Frostman transforms are Blaschke products.

\section{REFERENCES}

[A] A. B. Aleksandrov, The multiplicity of boundary values of inner functions (Russian), Izv. Akad. Nauk Armyan. SSR Ser. Mat. 22 (1987), 490-503. MR 89e:30058

[BCP] C. L. Belna, F. W. Carroll and G. Piranian, Strong Fatou-1-points of Blaschke products, Trans. Amer. Math. Soc. 280 (1983), 695-702. MR 85h:30039

[C] C. Carathéodory, Theory of Functions, Vol. II, Chelsea, New York, 1960.

[CM] J. A. Cima and A. L. Matheson, Essential norms of composition operators and Aleksandrov measures, Pacific J. Math. 179 (1997), 59-63. MR 98e:47047

[F] S. D. Fisher, Function Theory on Planar Domains, J. Wiley \& Sons, New York, 1983. MR 85d:30001 
[Fr] O. Frostman, Sur les produits de Blaschke, Kungl. Fysiog. Sällsk. i Lund Förh. 12 (1942), 169-182. MR 6:262e

[G] J. B. Garnett, Bounded Analytic Functions, Academic Press, New York, 1981. MR 83g:30037

[L] O. Lehto, A majorant principle in the theory of functions, Math. Scand. 1 (1953), 5-17. MR 15:115d

[Li1] J. E. Littlewood, On inequalities in the theory of functions, Proc. London Math. Soc. 23 (1925), 481-519.

[Li2] J. E. Littlewood, Lectures on the theory of functions, Oxford Univ. Press, Oxford, 1944. MR 6:261f

[N] R. Nevanlinna, Eindeutige analytische Funktionen, J. W. Edwards, Ann Arbor, Michigan, 1944. Second edition by Springer-Verlag, Berlin, 1953.

[Ra] T. Ransford, Potential Theory in the Complex Plane, Cambridge Univ. Press, Cambridge, 1995. MR 96e:31001

[R1] W. Rudin, A generalization of a theorem of Frostman, Math. Scand. 21 (1967), 136-173. MR 38:3463

[R2] W. Rudin, Real and Complex Analysis (3rd ed.), McGraw-Hill, New York, 1987.

[Sa1] D. Sarason, Composition operators as integral operators, Analysis and Partial Differential Equations, Marcel Dekker, New York, 1990. MR 92a:47040

[Sa2] D. Sarason, Sub-Hardy Hilbert Spaces in the Unit Disk, Wiley, New York, 1995. MR 96k:46039

[Sh] J. E. Shapiro, Aleksandrov measures used in essential norm inequalities for composition operators, J. Operator Theory 40 (1998), 133-146. MR 99i:47062

[S1] J. H. Shapiro, The essential norm of a composition operator, Ann. Math. 125 (1987), 375-404. MR 88c:47058

[S2] J. H. Shapiro, Recognizing an inner function by its distribution of values, unpublished manuscript, 1999. Available at

http://www.math.msu.edu/ shapiro/Pubvit/Downloads/InnerNev/InnerNev.html.

[SS] J. H. Shapiro and C. Sundberg, Compact composition operators on $L^{1}$, Proc. Amer. Math. Soc. 108 (1990), 443-449. MR 90d:47035

[St] C. S. Stanton, Counting functions and majorization for Jensen measures, Pacific J. Math. 125 (1986), 459-468. MR 88c:32002

Department of Mathematics, University of Helsinki, P.O. Box 4 (Yliopistonkatu 5), FIN-00014 University of Helsinki, Finland

E-mail address: pekka.j.nieminen@helsinki.fi

Department of Mathematics and Statistics, University of Jyväskylä, P.O. Box 35 (MAD), FIN-40014 University of JyvÄskylä, Finland

E-mail address: saksman@maths.jyu.fi 